\title{
Japanese encephalitis in children from an endemic district of West Bengal, India: A hospital based observational study
}

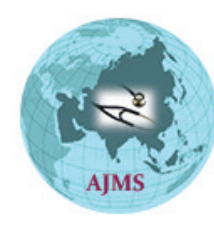

\author{
Abhishek Gupta', Sumanta Laha², Kanai Lal Barik ${ }^{3}$ \\ ${ }^{1}$ Junior Resident, ${ }^{2}$ Associate Professor, ${ }^{3}$ Professor, Department of Pediatric Medicine, Burdwan Medical College and \\ Hospital, East Burdwan, West Bengal, India
}

Background: Japanese encephalitis (JE) is an important cause of viral encephalitis in children in South East Asian countries including India. Aims and Objective: We have done this study in an JE endemic district of India to know the demographic profile, clinical presentation, seasonal variation, outcome and about the vaccination status of the JE cases. Materials and Methods: This observational, cross sectional study was done for a period of one year in the Pediatric department of Burdwan Medical College, West Bengal in children up to the age of 12 year, presented with acute encephalitis syndrome (AES). Demographic data, clinical presentation and JE vaccination status were recorded and confirmed JE cases were detected by either serum or CSF JE IgM antibody. Outcome of the JE cases were noted as discharge, death or leave against medical advice (LAMA). Results: We have found confirmed JE in 18 children out of total 125 AES cases $(14.4 \%) .61 .1 \%$ of them were within 6 to 12 year age, mostly from low socioeconomic status and $100 \%$ case occurred during monsoon and post monsoon period. History, was of JE vaccination were present in only $11.1 \%$ case of JE. Most common presenting symptoms were high fever $(100 \%)$ convulsion (94.4\%) and altered sensorium (72.2\%). $77.8 \%$ JE case were discharged and $11.1 \%$ expired. Conclusion: We must consider JE whenever a child from any endemic district comes with AES and we should try to bring every child under the JE vaccination coverage in the endemic regions globally to reduce the burden of this preventable encephalitis.
Access this article online

Website:

http://nepjol.info/index.php/AJMS DOI: $10.3126 /$ ajms.v12i10.37395

E-ISSN: 2091-0576

P-ISSN: $2467-9100$

Copyright (c) 2021 Asian Journal of Medical Sciences

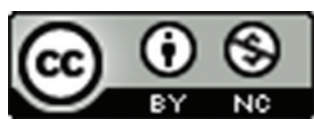

This work is licensed under a Creative Commons Attribution-NonCommercial 4.0 International License.

Key words: Japanese encephalitis, Children, West Bengal

\section{INTRODUCTION}

Japanese encephalitis virus (JEV) is a single stranded RNA virus of the flavivirus genus, same genus as of dengue or yellow fever viruses. It is transmitted by culex mosquito to human through a transmission cycle involving pig or water birds where human is the accidental dead end host. ${ }^{1} \mathrm{JEV}$ causes the disease called Japanese encephalitis (JE) named after its first documented case in Japan way back in $1871 .{ }^{2}$ Countries of South East Asian region including India are endemic for JE and it is one of the major cause of acute encephalitis syndrome (AES) in this region, with nearly 68000 clinical cases each year causing around 13000 to 20000 death. ${ }^{3}$ In India there is approximately 2500 clinical cases of JE and 500 death every year. ${ }^{4}$ West Bengal, Bihar, Uttar Pradesh, Karnataka, Tamil Nadu, Andhra Pradesh and Assam are the few examples of highly endemic states of India. ${ }^{5}$ First outbreak in India happened in Burdwan and Bankura district of West Bengal in the year 1973 with a repeat outbreak in Burdwan in 1976, after which a national level monitoring of Japanese encephalitis cases was started, integrated under N.M.E.P. (National Malaria Eradication Programme) from 1978. ${ }^{6}$ Since then Burdwan is among the 10 endemic districts of West Bengal, which is also famous as rice belt area, favourable for the transmission cycle of the virus. So Burdwan, our place of study historically and epidemiologically is important in the context of J.E. 
JE primarily affects the children and adolescents. Though it may be asymptomatic or mild in most cases, but serious disease can occur around 4 in 1000 infection with features of meningo-encephalitis causing case fatality rate as high as $30 \% .{ }^{2,3}$ At present there is no curable treatment for the disease and management is mainly symptomatic and supportive. But prevention may be possible and JE vaccination is ongoing under the national immunization programme in the endemic district of different states of India including the district of our study, that is Burdwan district.

With this background, we carried out this study with the specific objectives to find out the clinical presentation and sample positivity rate of Japanese encephalitis among pediatric acute encephalitis syndrome patients presented in our hospital. We also evaluated the demographic profile of our patients, seasonal variation of the disease and whether vaccination status influence the disease prevalence or not.

\section{MATERIALS AND METHODS}

This was a hospital based, observational, epidemiological, cross sectional study done for a period of one year from $1^{\text {st }}$ January 2018 to $31^{\text {st }}$ December 2018 in the department of Pediatric Medicine, Burdwan Medical College and Hospital, West Bengal, India. All children up to the age of 12 years coming to the department of Pediatrics, with acute encephalitis syndrome (AES) according to the Integrated Disease Surveillance Project (IDSP) guideline were included in the study. Here AES is defined as, a child of any age with acute onset of fever and any of the following a) change in mental status (confusion, disorientation, coma, inability to talk), b) new onset of seizures (excluding simple febrile seizures), c) other early clinical findings like an increase in irritability, somnolence or abnormal behavior greater than that seen with usual febrile illness. An informed written consent was taken from each parent of the admitted children before enrolling them in the study. Study was approved by Institutional Ethics Committee of Burdwan Medical College, Burdwan.

We collected the data by a predesigned questionnaire from parents regarding demographic profile like name, age, sex, district, religion, socioeconomic status by modified Kuppuswamy scale and also previous medical history and vaccination status particularly about JE vaccination. We have done thorough clinical examination and relevant laboratory examination needed for a case of AES. Two $\mathrm{ml}$ of venous blood was collected to test serum IgM antibody against JE by ELISA (enzyme-linked immunosorbent assay) method and cerebrospinal fluid (CSF) analysis was done for cytological, microbiological, biochemical and JEV IgM ELISA examination.
We used some operational definition in our study like a) sample positivity rate (SPR) which is the number of cases whose sample were found reactive for $\mathrm{JE} /$ number of cases whose sample were tested for JE $* 100$ and b) confirmed JE case as a suspected AES case confirmed by laboratory tests like serum or CSF JE IgM ELISA. Outcome of the confirmed JE cases were recorded as discharge, death or leave against medical advice (LAMA).

Statistical analysis was done using MedCalc statistical software, where we have done comparison of proportion analysis with chi squared test taking $\mathrm{p}$ value less than 0.05 as statistically significant. We have also calculated odds ratio (OR) with 95\% confidence interval where OR $>1$ indicates statistically significant association.

\section{RESULTS}

A total of 125 children with AES were included in the study out of which $72(58 \%)$ were males and $53(42 \%)$ females. We have found confirmed case of JE in 18 children by positive JE IgM antibody either in CSF or in serum. So, the sample positivity test (SPR) is $14.4 \%$ in our study.

Table 1 shows that number of JE cases are increasing from lower to higher age group. It is $11.1 \%$ below 1 year,27.8\% between 1 to 6 years and $61.1 \%$ above byears. The difference is statistically significant between 6 to 12 year age group with other two age groups $(\mathrm{p}<0.05)$. JE cases were equally distributed among male and female child (50\% each) in our study. Among the 18 children with JE, one each from upper and upper middle class, 10 from lower middle, 4 from upper lower and 2 from lower class according to the modified Kuppuswami scale. So, the disease is definitely more prevalent in low socioeconomic class. When we

\begin{tabular}{lcc}
$\begin{array}{l}\text { Table 1: Demographic profile and seasonal } \\
\text { variation of JE cases }\end{array}$ & $\begin{array}{l}\text { Number of } \\
\text { subject (N=18) }\end{array}$ & Percentage(\%) \\
\hline Parameter & 2 & 11.1 \\
& 5 & 27.8 \\
\hline Age( in Years) & 11 & 61.1 \\
$\quad<1$ & & 50 \\
1 to 6 & 9 & 50 \\
6 to 12 & 9 & 5.6 \\
Sex & & 61.1 \\
$\quad$ Male & 1 & \\
Female & $11(1+10)$ & 33.3 \\
Socio-economic status & & \\
$\quad$ Upper(I) & $6(4+2)$ & 0 \\
Middle(Upper middle,II & & 100 \\
$\quad$ \& lower Middle,III) & & \\
$\quad$ Lower(Upper lower,IV & 0 & \\
$\quad$ L Lower,V) & 0 & \\
Month wise case & $18(1+5+6+6)$ & \\
$\quad$ December to March & & \\
$\quad$ April to July & August to November &
\end{tabular}


observe the seasonal pattern of JE case, there is no case from December to July, 1 case in August, 5 in September and 6 each in October and November. So, there is cluster of cases from August to November, that is in monsoon and post monsoon period.

Table 2 shows the major clinical feature with which a case of JE presented in our hospital. There is definitely overlapping clinical presentation, but all $18(100 \%)$ children had moderate to high grade of fever for last few days at the time of admission. Among them 17(94.4\%) had seizure, $13(72.2 \%)$ had some kind of altered sensorium, $8(44.4 \%)$ had signs of meningeal irritation and $7(38.9 \%)$ children had headache with vomiting.

Table 3 shows that out of $18 \mathrm{JE}$ patients 14 (77.8\%) were discharged from our hospital, 2 patients leave against medical advice and 2 patients (11.1\%) expired after all effort including ventilator support. But still we were able to discharge a significant proportion of JE patients from our setup $(\mathrm{p}<0.05)$.

From table 4, we can see that only $11.1 \%$ (2 out of 18 ) case of JE had a history of JE vaccination with a significant proportion $88.9 \%$ (16 out of 18 ) were not vaccinated with even a single dose of JE vaccine $(\mathrm{p}<0.05)$. Percentage of JE case was higher among non-vaccinated (16/42 i.e., 38\%) than vaccinated (2/83 i.e., $2.4 \%)$ child. If we compare JE and non-JE AES with their vaccination status, we found the odds ratio more than 1 , which means non vaccination against $\mathrm{JE}$ is a risk factor, that is positively associated with

\section{Table 2: Major Clinical presentation of JE cases}

\begin{tabular}{lcc} 
Clinical feature & No of case(N=18) & Percentage (\%) \\
\hline Fever & 18 & 100 \\
Convulsion & 17 & 94.4 \\
Altered mental status & 13 & 72.2 \\
Positive meningeal sign & 08 & 44.4 \\
Headache with vomiting & 07 & 38.9 \\
\hline
\end{tabular}

\section{Table 3: Outcome of JE cases}

\begin{tabular}{lcc}
\hline Outcome & No of case(N=18) & Percentage \\
\hline Discharged & 14 & 77.8 \\
LAMA & 02 & 11.1 \\
Death & 02 & 11.1 \\
\hline
\end{tabular}

\begin{tabular}{|c|c|c|c|}
\hline $\begin{array}{l}\text { Vaccination } \\
\text { status }\end{array}$ & $\frac{\text { Total case }}{(n=125)}$ & $\frac{\text { JE case }}{(n=18)}$ & $\frac{\text { AES other than JE }}{(n=107)}$ \\
\hline $\begin{array}{l}\text { Not Vaccinated } \\
\text { JE Vaccinated }\end{array}$ & $\begin{array}{l}42 \\
83\end{array}$ & $\begin{array}{l}16 \\
02\end{array}$ & $\begin{array}{l}26 \\
81\end{array}$ \\
\hline
\end{tabular}

the occurrence of the disease. (odds ratio 24.9 with $95 \%$ CI 5.37 to 115.67$)$.

\section{DISCUSSION}

In our study, the sample positivity rate (SPR) of JE was $14.4 \%$ in children up to 12 years of age which is in corroborative to the study conducted by Chakraborty $\mathrm{D}$ et al., in 2015 who found SPR $11.23 \%$ in children up to 10 years of age. ${ }^{7}$ But the other studies done before showed quite higher SPR for JE infection. Study conducted by Sarkar A et al., (2005 - 2010) found it 27\%, S K Anuradha et al., 23.17\%, Bandhopadhyay B et al., 22\% (2011) and Kakoti G et al., (2013)30\%.8.11 On the contrary, one study conducted by Gunasekaran et al. (2007-09) revealed SPR as low as $4.9 \% .{ }^{12}$ It appears that the percentages of Japanese encephalitis positive cases were decreased after 2013 due to the better awareness programs, extensive vaccination against JE in the endemic districts with live attenuated JE vaccine SA-14-14-2., or natural epidemiological shift due to herd immunity. Cross-protection by other flaviviral diseases, like dengue, which is in the rise in recent years due to increased urbanization may be another reason for decline of the JE cases to some extent. The significant decline of JE cases in our study could be attributed to all these factors.

Even though JE cases were found even in infancy in this study, but it is particularly prevalent in the age group of 6 to 12 years. This increased incidence of JE among children and adolescents is often due to their visit in the paddy fields with their parents to help them in the process of cultivation thus exposing themselves to the bite of culex mosquito.Other studies like Anuradha et al.(most cases in 7- 9 years), Kakoti G et al., (most cases between 5-12 year) shows similar results like our study., ${ }^{911}$ In contrast study by Bista $\mathrm{MB}$ et al showed more incidence of JE cases in age group between 1-5 years than 5-9 years. ${ }^{13}$

Although SPR was more in females $(9 / 53$ or $16.9 \%)$ as compare to male $(9 / 72$ or $12.5 \%)$ cases admitted with AES in our institute, but the incidence of JE case is equal in both the sexes (9 each). Studies like Shrestha M et al., also showed male predominance, which presumably because of increased involvement of adolescent male in the areas of rice cultivation. ${ }^{14}$ There was no sex predilection among the JE cases in study in 2010 done by Sarkar et al., which is concurring with our study. ${ }^{8}$

In this study, it is seen that 16 out of $18 \mathrm{JE}$ case are from stage III, IV, V (lower middle, upper lower and lower) of modified Kuppuswamy scale 2017. That means almost 88\% 
patients of JE belong to middle or low socioeconomic class. This finding correlates from other studies like Kakoti G et al., Potula R et al., or Kumari R et al., where they found majority of the patients coming with JE were from the rural area and belonged to low socioeconomic group and engaged in the cultivation in paddy fields. ${ }^{11,15,16}$

Our study shows a seasonal variation in the incidence of JE cases. We have divided the season into three group, winter and spring (December to March), summer and early monsoon (April to July) and late monsoon and early winter (August to November). It is seen that there is no case from December to July, one case in August and rest of the JE cases are concentrated between September to November. Other studies like Anuradha et al., Sarkar et al., and Bista et al., have also reported higher incidence of JE during similar months due to increased prevalence of the vector mosquitoes Culex, which breed abundantly in the paddy fields covered with stagnant water during and after the rainy season. ${ }^{8,9,13}$ In our study, epidemic curve of JE cases showed a distinct peak in the late monsoon and early winter. In the 6 districts of northern part of West Bengal, cases reached peak in the month of August followed by a sharp decline. ${ }^{8}$ Study of Bandhopadhyay B et al., revealed an upsurge of cases during and after the rainy season increasing from August and peak in November. ${ }^{10}$

JE infection may be asymptomatic or just a mild febrile illness with headache in most of the cases. But approximately 4 in 1000 infections is resulted in a stormy course with high case fatality rate. ${ }^{3}$ After 4-14 days incubation period, it progress through the following 4 stages: prodromal phase (2-3 days), acute stage (3-4 days), sub acute stage (710 days), and convalescence (4-7 wk). In our study most of the patients presented in acute phase with mixed feature of high-grade fever, altered sensorium, generalized convulsion associated with headache and neck rigidity. Many of them had a history of gastrointestinal symptoms like vomiting, loose stool and abdominal pain in the prodromal phase. Same clinical manifestations are noted by other studies like study of Prafulla Datta. ${ }^{11}$ Few previous studies also found spastic paralysis and pronounced extra pyramidal signs which is not the presenting feature in our study.

In the present study we have successfully discharged $77.8 \%$ (14 out of 18) patients. Among the rest, two patients leave our facility in the subacute phase as the poor family cannot stay in the hospital during the harvesting season and two died. So, the case fatality rate in our institute is $11.1 \%$, which may be due to prompt diagnosis, early management and pediatric intensive care unit facility. Other studies like Kakoti $G$ et al., shows almost similar case fatality rate as $14.7 \%{ }^{11}$ Study of vaccination status of the affected children revealed some important findings. Present study showed that among the JE cases, only $11 \%$ were vaccinated with either one or two doses of JE vaccine and the rest $89 \%$ were not vaccinated. In our study it is seen that non vaccinated children are definitely at higher risk for developing JE (odds ratio $>1$ ) than their vaccinated counterpart. JE vaccination was first started in India in 2006 with a single dose of Chinese live attenuated vaccine strain SA-14-14-2 $\mathrm{JE}$ vaccine in children of 1 to 15 years. The same vaccine has been included in routine immunization under Universal Immunization Programme (UIP) in endemic states of India including West Bengal as a single dose at 16-18 months with DPT booster in $2011 .{ }^{17}$ In 2013, another dose of the vaccine was added at 9 months age along with measles vaccine. Being a endemic district in West Bengal, Burdwan is also following the same immunization schedule but still could not cover the target children sufficiently. Though the protective efficacy of JE vaccines varies in different study but it has a definite role in preventing the disease. A study by Babasaheb V Tandale et al., in 2018 shows a $72.2 \%$ protective efficacy with any dose of the JE vaccine. ${ }^{18}$ Our study also shows that proportion of JE cases is higher among non vaccinated than the vaccinated AES case.

\section{CONCLUSION}

We should suspect Japanese encephalitis in every child presented with AES from an endemic district of any country all over the world. In our study we have found JE more prevalent in the 6to12 year age group and mainly in the low socioeconomic class. It is presented mostly with high fever, convulsion and altered sensorium with a seasonal variation showing clustering of cases during the monsoon and post monsoon period. As failure to take even a single dose of JE vaccine may act as risk factor for JE, so it is extremely important to bring all the children under the JE vaccination coverage in the endemic region of the state and country to reduce the mortality and morbidity related to this vaccine preventable encephalitis.

\section{ACKNOWLEDGEMENT}

We express our sincere thanks to all the members of the Department of Pediatrics, Burdwan Medical College for their constant support and encouragement in the successful completion of the study.

\section{REFERENCES}

1. CDC. Transmission of Japanese Encephalitis Virus, Japanese Encephalitis, CDC: https://www.cdc.gov/JapaneseEncephalitis/ transmission/index.html

2. Center for Disease Control and Prevention (CDC), "Question and answer about Japanese Encephalitis," March 2013, http:// 
www.cdc.gov/japaneseencephalitis/qa/index.html

3. Japanese encephalitis. World Health Organization, 2019 report. https://www.who.int/news-room/fact-sheets/detail/japanese encephalitis

4. NVBDC P. Directorate General of Health Services. Ministry of Health and Family Welfare. New Delhi. 2010. http://nvbdcp.gov. in/je-cd.html

5. Kulkarni R, Sapkal GN, Kushal H and Mourya DT. Japaneese Encephalitis: A brief review on Indian perspectives. Open Virol J. 2018; 12:121-130.

https://doi.org/10.2174/1874357901812010121

6. Chakravarty SK, Sarkar JK, Chakravarty MS, Mukherjee MK, Mukherjee KK, Das BC, et al. The first epidemic of Japanese encephalitis studied in India- virological studies. Indian J Med Res.1975; 63(1):77-82.

7. Chakraborty D, Banerjee S, Maji D, Dey TK, Mondal P and Basu M. A Descriptive Study of Japanese Encephalitis in West Bengal, India, Based on Surveillance Data: Changing Pattern Observed in Recent Years. Sch J App Med Sci.2015;3(1E):320-328.

8. Sarkar A, Taraphdar D, Mukhopadhyay BB, Kumar M, Mukhopadhyay SK and Chatterjee S. Influence of socioeconomic status and environmental factors on serologically diagnosed Japanese encephalitis cases in the state of West Bengal, India during 2005-2010. Health. 2012. 14(1): 6-12. https://doi.org/10.4236/health.2012.41002

9. Anuradha SK, Surekha YA, Sathyanarayan MS, Suresh S, Satish P, Mariraj J, et al. Epidemiological aspects of Japanese encephalitis in Bellary, Karnataka, India. International Journal of Biological and Medical Research.2011;2(3):691-695.

10. Bandyopadhyay B, Bhattacharyya I, Adhikary $S$ et al. Incidence of Japanese Encephalitis among Acute Encephalitis Syndrome cases in West Bengal,India. BioMed Research International, 2013, Article ID 896749, 5 pages. http://.doi. org/10.1155/2013/896749

https://doi.org/10.1155/2013/896749
11. Kakoti G, Dutta P, Das BR, Borah J and Mahanta J. Clinical Profile and Outcome of Japanese Encephalitis in Children Admitted with Acute Encephalitis Syndrome. BioMed Research International, 2013, Article ID 152656, 5 pages. http://dx.doi. org/10.1155/2013/152656 https://doi.org/10.1155/2013/152656

12. Gunasekaran P, Kaberi K, Arunagiri K, Mohana S, Kiruba R, Senthil Kumar V, et al. Japanese Encephalitis in Tamil Nadu (2007-2009). Indian J Med Res.2012;135:680-682.

13. Bista MB and Shrestha JM. Epidemiological situation of Japanese Encephalitis in Nepal. J Nepal Med Assoc.2005;44(158):51-56. https://doi.org/10.31729/jnma.397

14. Shrestha M, Gupta R and Gautam I. Epidemio-entomological study of Japanese encephalitis in Bhaktapur District. Nepal. J Inst Sci Technol. 2015;20(1):112-118. https://doi.org/10.3126/jist.v20i1.13932

15. Potula R, Badrinath $S$ and Srinivasan $S$. Japanese encephalitis in and around Pondicherry, south India: a clinical appraisal and prognostic indicators for the outcome. Journal of Tropical Pediatrics.2003;49(1):48-53. https://doi.org/10.1093/tropej/49.1.48

16. Kumari R and Joshi PL. A review of Japanese encephalitis in Uttar Pradesh, India. WHO South East Asia Journal of Public Health. 2012;1(4):374-395. https://doi.org/10.4103/2224-3151.207040

17. Programme Implementation Plan 2011-12; National Rural Health Mission/Health and Family Welfare Department, West Bengal. Disease control programme.http://pipnrhmmohfw.nic.in/ index_files/non_high_focus_large/westbengal/4d.pdf

18. Tandale BV, Khan SA, Kushwaha KP, Rahman $\mathrm{H}$ and Gore MM. Effectiveness of Japanese Encephalitis SA 14-142 live attenuated vaccine among Indian children: Retrospective 1:4 matched case control study. J Infect Public Health. 2018;11(5):713-719. https://doi.org/10.1016/j.jiph.2018.04.011

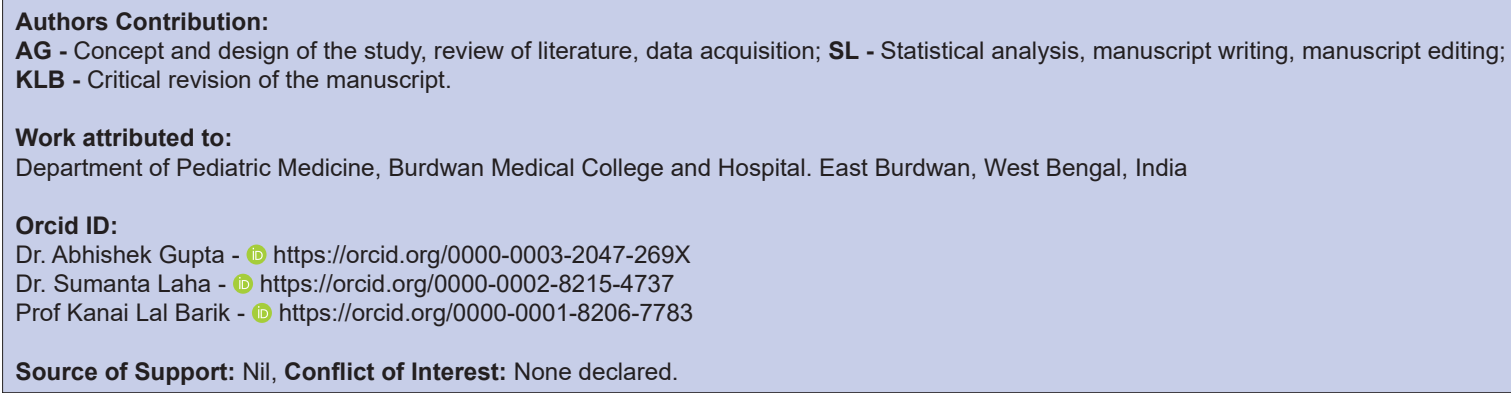

